\title{
How Progesterone Impairs Memory for Biologically Salient Stimuli in Healthy Young Women
}

\author{
Guido van Wingen, ${ }^{1,2}$ Frank van Broekhoven, ${ }^{2}$ Robbert Jan Verkes, ${ }^{2}$ Karl Magnus Petersson, ${ }^{1}$ Torbjörn Bäckström, ${ }^{4}$ \\ Jan Buitelaar, ${ }^{2}$ and Guillén Fernández ${ }^{1,3}$ \\ ${ }^{1}$ F. C. Donders Centre for Cognitive Neuroimaging, Radboud University Nijmegen, 6525 EN Nijmegen, The Netherlands, Departments of 2Psychiatry and \\ ${ }^{3}$ Neurology, Radboud University Nijmegen Medical Centre, 6525 GC Nijmegen, The Netherlands, and ${ }^{4}$ Umeå Neurosteroid Research Center, Department of \\ Clinical Science, Obstetrics, and Gynecology, Norrlands University Hospital, SE 90185 Umeå, Sweden
}

Progesterone, or rather its neuroactive metabolite allopregnanolone, modulates amygdala activity and thereby influences anxiety. Cognition and, in particular, memory are also altered by allopregnanolone. In the present study, we investigated whether allopregnanolone modulates memory for biologically salient stimuli by influencing amygdala activity, which in turn may affect neural processes in other brain regions. A single progesterone dose was administered orally to healthy young women in a double-blind, placebo-controlled, crossover design, and participants were asked to memorize and recognize faces while undergoing functional magnetic resonance imaging. Progesterone decreased recognition accuracy without affecting reaction times. The imaging results show that the amygdala, hippocampus, and fusiform gyrus supported memory formation. Importantly, progesterone decreased responses to faces in the amygdala and fusiform gyrus during memory encoding, whereas it increased hippocampal responses. The progesterone-induced decrease in neural activity in the amygdala and fusiform gyrus predicted the decrease in memory performance across subjects. However, progesterone did not modulate the differential activation between subsequently remembered and subsequently forgotten faces in these areas. A similar pattern of results was observed in the fusiform gyrus and prefrontal cortex during memory retrieval. These results suggest that allopregnanolone impairs memory by reducing the recruitment of those brain regions that support memory formation and retrieval. Given the important role of the amygdala in the modulation of memory, these results suggest that allopregnanolone alters memory by influencing amygdala activity, which in turn may affect memory processes in other brain regions.

Key words: fMRI; progesterone; allopregnanolone; memory; emotion; amygdala

\section{Introduction}

Changes in gonadal steroid hormone levels are associated with changes in mood regulation (Steiner et al., 2003) and cognition (Kimura, 1999). For example, the highest physiological levels of progesterone and estradiol are reached during pregnancy, which is accompanied by a worsening of mood and a decrease in memory performance (Sharp et al., 1993; Keenan et al., 1998; Buckwalter et al., 1999; de Groot et al., 2003; Bennett et al., 2004). The more modest hormone level changes during the menstrual cycle have more subtle and variable effects on mood and cognition in healthy women (Hampson, 1990; Phillips and Sherwin, 1992; Sveindottir and Backstrom, 2000; Maki et al., 2002) but induce negative mood symptoms and impair memory in women with premenstrual syndrome (Schmidt et al., 1998; Schmitt et al., 2005). These effects are potentially mediated by the neuroactive metabolites of progesterone. The metabolite allopregnanolone

Received April 17, 2007; revised Sept. 5, 2007; accepted Sept. 5, 2007.

This work was supported by an internal grant from the Radboud University Nijmegen Medical Center, a European Union structural fund objective 1 program, and Swedish Research Council Project 11198. We thank Vera Dinkelacker for providing part of the stimulus material.

Correspondence should be addressed to Guido van Wingen, F. C. Donders Centre for Cognitive Neuroimaging, Kapittelweg 29, 6525 EN Nijmegen, The Netherlands. E-mail: guido.vanwingen@fcdonders.ru.nl.

DOI:10.1523/JNEUROSCI.1715-07.2007

Copyright $\odot 2007$ Society for Neuroscience $\quad 0270-6474 / 07 / 2711416-08 \$ 15.00 / 0$ potentiates the inhibitory effect of GABA by modulating the $\mathrm{GABA}_{\mathrm{A}}$ receptor (Majewska et al., 1986) and has been suggested to mediate the negative effects of progesterone on mood $(\mathrm{N}$ Wihlbäck et al., 2006) and memory (Brett and Baxendale, 2001). Although allopregnanolone can also induce paradoxical effects at moderate concentrations (Fish et al., 2001; Andréen et al., 2006; van Wingen et al., 2007), animal studies suggest that allopregnanolone usually decreases anxiety (Bitran et al., 1991, 1995; Wieland et al., 1991) and impairs memory (Ladurelle et al., 2000; Johansson et al., 2002), suggesting a possible relationship between the effects of allopregnanolone on emotion and memory.

Allopregnanolone induces its anxiolytic effect by action on the amygdala (Akwa et al., 1999), a brain structure that is known to influence various cognitive processes during emotional experiences, including memory formation (Cahill et al., 1996; Canli et al., 2000). Animal studies have shown that the amygdala mediates the mnemonic effects of GABAergic drugs (Brioni et al., 1989). Specifically, the basolateral amygdala appears critical in mediating the amnesic effects of benzodiazepines (Tomaz et al., 1992). Hence, a modulation of basolateral amygdala activity by progesterone and/or allopregnanolone, with putative indirect effects on other brain regions such as the hippocampus (Richardson et al., 2004) or the inferior temporal cortex (ITC), might link hormone action with memory performance. 
To test this hypothesis, we combined a single progesterone administration to healthy young women with functional magnetic resonance imaging (fMRI) in a double-blind, placebocontrolled, crossover design. During scanning, participants were asked to memorize and recognize faces. The perception of faces elicits amygdala activity (Somerville et al., 2004; Fitzgerald et al., 2006), and the amygdala modulates the neural response to faces in the fusiform gyrus (Vuilleumier et al., 2004). This brain region is preferentially involved in face processing (Kanwisher et al., 1997) and presumably involved in face memory storage (Miyashita, 1993). Whereas the amygdala modulates memory consolidation in other brain regions (McGaugh, 2004), the prefrontal cortex exerts executive control during memory retrieval of visual representations in the ITC (Tomita et al., 1999). Therefore, we hypothesized that allopregnanolone impairs memory for faces by modulating ITC activity, potentially by modulating amygdala activity during encoding and prefrontal activity during retrieval.

By adopting a factorial event-related fMRI design, we are able to identify the mechanism by which allopregnanolone modulates memory formation and retrieval. Initially, we identified brain regions supporting memory by comparing neural responses during study and test to items remembered or forgotten on the (subsequent) memory test (i.e., main effect of memory). If allopregnanolone impairs memory performance by altering these neural processes, it would change the differential activation between (subsequent) hits and (subsequent) misses, as reflected by a significant drug $\times$ memory interaction. Conversely, allopregnanolone may not change these memory processes per se, but it may impair memory performance by altering the recruitment of these neural processes (cf. Uncapher and Rugg, 2005). Allopregnanolone would then reduce the response amplitudes to both (subsequent) hits and (subsequent) misses within those brain regions that support memory, as reflected by a significant conjunction between the main effects of drug and memory.

\section{Materials and Methods}

Participants. Eighteen women with a mean age of 24 years (range, 19-39) gave written informed consent to participate in this study, which had been approved by the local medical ethics committee (CMO Regio Arnhem-Nijmegen, The Netherlands). The participants were healthy as determined by routine physical and laboratory examinations, had no current neurological or psychiatric disorder as measured by a structured interview [MINI (Mini-International Neuropsychiatric Interview)] (Sheehan et al., 1998), and reported no history of psychiatric or somatic disease potentially affecting the brain. They were right handed, free of medication, did not use hormonal contraceptives, and had a regular menstrual cycle. Data of one subject were lost because of a technical failure, and data of another were excluded because of chance-level recognition performance. Therefore, the results are based on the data of 16 participants.

Study design and procedure. Subjects participated twice during the early follicular phase (days 2-7) of different menstrual cycles to ensure low endogenous gonadal steroid hormone levels. The estimated median duration between sessions was 2.1 (interquartile range, 1.9) menstrual cycles. They arrived at $\sim 8: 15$ A.M. after an overnight fast to receive a standardized light breakfast, after which $400 \mathrm{mg}$ of micronized progesterone or placebo was administered, in a double-blind, crossover manner. The progesterone dose was administered orally, to increase the allopregnanolone concentration to a similar extent (de Lignieres et al., 1995). Administration was randomized among pairs of subjects, to ensure that the order of versions of the memory paradigm was the same per subjectpair (see below). A venous blood sample was collected before drug administration, before scanning, and after scanning $(\sim 0,80$, and $190 \mathrm{~min}$ relative to the drug administration). In addition, subjects completed the mood rating scale (MRS) (Bond and Lader, 1974) before scanning and the state version of the state trait anxiety inventory (STAI) (Spielberger et al., 1970) after scanning. The MRS contains 16 visual analog scales that measure the three principal components alertness (scoring range, $0-625.2)$, contentedness $(0-352.9)$, and calmness $(0-152.2)$. The STAI is a 20 -item, four-point Likert scale that measures state anxiety $(20-80)$.

Serum analysis. Progesterone was measured with Delfia progesterone kits (Wallac, Turku, Finland) according to the manufacturer's instructions. Allopregnanolone was measured with a radioimmunoassay after diethylether extraction and Celite chromatography (recovery, 78\%). The antiserum was raised against $3 \alpha$-hydroxy-20-oxo- $5 \alpha$-pregnan-11-yl carboxymethyl ether-BSA (a gift from Dr. R. H. Purdy, College of Medicine, University of California San Diego, La Jolla, CA) with low crossreactivity. The intra-assay coefficient of variation was $6.5 \%$, and the inter-assay coefficient of variation was $8.5 \%$ (Andréen et al., 2005).

Memory paradigm. The subjects completed four study-test cycles during scanning. During study, subjects had to memorize pictures of male and female faces, while making a gender decision. During test, they had to recognize these pictures among a series of new pictures and to make an old, new, or unsure decision. For three subjects, only two study-test cycles were available for one session, in which case only the respective cycles from the other session were used.

The stimuli consisted of faces with a neutral to mildly happy emotional expression and with direct gaze direction. They were derived from different stimulus sets (Ekman and Friesen, 1976) [the Karolinska Directed Emotional Faces (1998; D. Lundqvist, A. Flykt, and A. Öhman, Karolinska Institute, Stockholm, Sweden) and the AR face database (1998; A. Martinez and R. Benavente, Purdue University, West Lafayette, IN)] and the internet. All photos were edited (Photoshop 6.0; Adobe Systems, San Jose, CA) to produce oval grayscale pictures that showed the face from chin to forehead and excluded the ears. The 960 stimuli were divided over eight blocks of 120 faces each, which did not differ in the amount of features (50\% male, $24 \%$ glasses, $6 \%$ facial hair, and $4 \%$ non-Caucasian; $\chi^{2}(21)=1.5$, NS). Half of the stimuli per block were used as study items, and half were used as distractors during test. In addition, half of the blocks were used per session. These two factors were counterbalanced over subject-pairs. The stimuli were pseudo-randomly intermixed with 10 null events ( $2 \mathrm{~s}$ ) per study and per test phase, such that there were no more than four consecutive presentations per gender or old/new status of the stimulus. All faces were presented for $0.8 \mathrm{~s}$ with an interstimulus interval of 3.3-4.3 s. The discrimination index $d^{\prime}$ was used as a measure of recognition accuracy, which was calculated as the $z$ score of the hit rate minus the $z$ score of the false-alarm rate (Hochhaus, 1972). The hit and false-alarm rates were defined as the number of hits or false alarms divided by the number of old or new trials for which an old or new decision was made.

Image acquisition. MR data were acquired with a $1.5 \mathrm{~T}$ Siemens (Erlangen, Germany) Sonata MR scanner, equipped with a standard head coil. Two runs of $854 \mathrm{~T}^{*}$-weighted blood oxygenation level-dependent (BOLD) images were acquired using echo-planar imaging (EPI) with each image volume consisting of 33 axial slices [ $3 \mathrm{~mm}, 0.5 \mathrm{~mm}$ slice gap; repetition time (TR), $2.290 \mathrm{~s}$; echo time (TE), $30 \mathrm{~ms} ; 64 \times 64$ matrix; field of view (FOV), $224 \mathrm{~mm}$; flip angle, $90^{\circ}$ ]. In addition, a high-resolution T1-weighted structural MR image was acquired for spatial normalization procedures [3D MP-RAGE (magnetization-prepared rapid gradientecho); TR, $2250 \mathrm{~ms}$; TE, $3.93 \mathrm{~ms}$; 176 contiguous $1 \mathrm{~mm}$ slices; $256 \times 256$ matrix; FOV, $256 \mathrm{~mm}$ ].

Image analysis. Image analysis was performed with SPM2 (Wellcome Department of Imaging Neuroscience, London, UK). The first five EPI volumes were discarded to allow for $\mathrm{T} 1$ equilibration, and the remaining images were realigned to the first volume. Images were then corrected for differences in slice acquisition time, spatially normalized to the Montreal Neurological Institute (MNI) T1 template, super-sampled into $2 \times 2 \times 2$ $\mathrm{mm}^{3}$ voxels, and spatially smoothed with a Gaussian kernel of $10 \mathrm{~mm}$ full-width at half-maximum.

Statistical analysis was performed within the framework of the general linear model (Friston et al., 1995). Later remembered and later forgotten stimuli were separately modeled for the study phase, as were hits, misses, correct rejections, and false alarms for the test phase. Stimuli with an incorrect gender decision or omission during study, or with an unsure 
Table 1. Serum concentrations of progesterone and allopregnanolone after the oral administration of $400 \mathrm{mg}$ of micronized progesterone ( $\mathrm{nmol} / \mathrm{L}$ )

\begin{tabular}{lcc}
\hline Time (min) & Progesterone & Allopregnanolone \\
\hline 0 (baseline) & $1.9 \pm 0.3$ & $0.6 \pm 0.1$ \\
80 (before fMRI session) & $16.2 \pm 7.3$ & $23.3 \pm 8.4$ \\
190 (after fMRI session) & $174.2 \pm 68.4$ & $185.8 \pm 26.6$ \\
\hline
\end{tabular}

Data are mean \pm SEM.

response or omission during test, were included in a condition of no interest. The explanatory variables $(0.8 \mathrm{~s})$ and null events $(2 \mathrm{~s})$ were temporally convolved with the canonical hemodynamic response function of SPM2. In addition, the realignment parameters were included to model potential movement artifacts as well as a high-pass filter (cutoff at $1 / 128 \mathrm{~Hz}$ ). To account for various global effects, the EPI data were proportionally scaled. Temporal autocorrelation was modeled with an $\mathrm{AR}(1)$ process, and the parameter estimates were obtained by maximum likelihood estimation (Friston et al., 2002) to allow for departures from sphericity. The relevant parameter images contrasting each condition to null events were entered in a random-effects repeated-measures ANOVA with a nonsphericity correction. Statistical tests were family-wise error rate corrected for multiple comparisons across the entire brain. A small volume correction was used to correct for multiple comparisons across the search volume for regions of interest (Worsley et al., 1996). Because the basolateral amygdala mediates the amnesic effects of diazepam (Tomaz et al., 1992), the search volume for the amygdala was defined as a sphere with $7 \mathrm{~mm}$ radius around the probabilistic cytoarchitectonic center of the basolateral amygdala $[(-26,-8,-18)$ and $(28,-8,-18)]$, which approximates total amygdala volume (Amunts et al., 2005; Eickhoff et al., 2005). Correspondingly, the hippocampal search volume was defined as a sphere with $10 \mathrm{~mm}$ radius around the center of the Cornu ammonis $[(-28,-28,-8)$ and $(28,-26,-8)]$. In addition, the faceresponsive region in the fusiform gyrus was defined as a sphere with 10 $\mathrm{mm}$ radius around previously reported Talairach coordinates (Kanwisher et al., 1997), which were transformed into MNI space [http:// imaging.mrc-cbu.cam.ac.uk/imaging/MniTalairach; $(-36,-64,-16)$ and $(40,-56,-16)]$. To exclude a general effect of the drug administration on the BOLD response, the primary visual cortex (V1) was used as the control region, which was defined analogously to the hippocampus (Amunts et al., 2000). The maxima of significant clusters are reported in MNI coordinates.

Hypothesis testing. Statistical tests were performed separately for encoding and retrieval. The main effects of drug and memory and the drug $\times$ memory interaction were estimated by contrasting the relevant task conditions. The main effects of memory were investigated by comparing neural responses for subsequent hits and subsequent misses during encoding and for hits and misses during retrieval, collapsed across drug conditions. The main effects of drug were investigated by comparing the responses for all trials versus null events between drug conditions. In addition, conjunction analyses were performed that tested the global null hypothesis using the minimum $T$ statistic as implemented within SPM2, to confirm the regional overlap between the main effects of memory and drug (Friston et al., 2005). To assess the relationship between neural activity and memory performance across subjects, mean activity within each significant conjunction cluster ( $p<0.001$, uncorrected) was extracted (Brett et al., 2002), and the differences in activity between the progesterone and placebo conditions were entered in regression analyses through the origin as a predictor for the difference in memory performance.

\section{Results}

\section{Serum concentrations and questionnaires}

The progesterone administration increased both the serum progesterone and allopregnanolone concentrations (condition $\times$ time interaction; progesterone: $F_{(2,12)}=5.8, p<0.05$; allopreganolone: $F_{(2,12)}=23.9, p<0.001$ ) (Table 1). As expected, the baseline concentrations were not significantly different between the progesterone and placebo sessions (progesterone: $t_{(15)}=0.6$, $p>0.5$; allopregnanolone: $\left.t_{(15)}=1.6, p>0.1\right)$. The progesterone administration produced serum concentrations of allopregnanolone that are similar to those reached during pregnancy (Luisi et al., 2000). However, progesterone had no significant effects on alertness [progesterone (mean \pm SEM), $410.1 \pm 19.3$; placebo, $\left.417.2 \pm 12.9 ; t_{(15)}=0.3 ; p>0.7\right]$, contentedness (progesterone, $271.4 \pm 8.7$; placebo, $273.7 \pm 7.2 ; t_{(15)}=0.4 ; p>0.7$ ), and calmness (progesterone, $108.9 \pm 5.3$; placebo, $115.2 \pm 3.6$; $\left.t_{(15)}=1.5 ; p>0.1\right)$ as measured with the MRS before scanning, nor on state anxiety (STAI; progesterone, $32.6 \pm 1.5$; placebo, $\left.33.8 \pm 1.0 ; t_{(15)}=0.7 ; p>0.4\right)$ after the scanning session, although the allopregnanolone concentration after scanning was in the range that could induce sedation (Timby et al., 2006). Differences in behavioral performance and neural activations can therefore not be explained by effects of progesterone on anxiety or mood states.

\section{Progesterone decreases memory performance}

The participants were asked to make a gender decision during the study phase of the memory task. Progesterone did not significantly affect the decision accuracy or reaction times [accuracy and reaction times (mean \pm SEM); progesterone: $93.8 \pm 0.8 \%, 1051 \pm 61 \mathrm{~ms}$; placebo: $94.3 \pm 1.2 \%, 1102 \pm 68$ $\mathrm{ms}$; both $p>0.3$ ), again indicating that progesterone was not sedative.

Critically, progesterone decreased the recognition accuracy for faces during the test phase of the memory task $\left(d^{\prime}\right.$; progesterone, $0.75 \pm 0.06$; placebo, $0.87 \pm 0.09 ; t_{(15)}=2.2 ; p<$ $0.05)$. The hit and false-alarm rates during the progesterone condition were $66.1 \pm 3.1$ and $38.3 \pm 2.8 \%$, respectively, and $68.6 \pm 2.2$ and $36.4 \pm 3.6 \%$ during the placebo condition. Reaction times during the test phase were fastest for hits and slowest for misses (hits $<$ false alarms $<$ correct rejections $<$ misses; $F_{(3,13)}=8.9 ; p<0.005$; all paired $t$ tests, $\left.p<0.005\right)$, but the reaction times were not significantly affected by progesterone (hits, false alarms, correct rejections, misses; progesterone: $1319 \pm 36,1396 \pm 42,1509 \pm 54$, and $1588 \pm 72$ ms; placebo: $1362 \pm 51,1457 \pm 56,1531 \pm 60$, and $1624 \pm 72$ $\mathrm{ms}$; all $F \leq 1$ ). In summary, the behavioral results show that progesterone specifically decreased recognition memory accuracy, without affecting other behavioral measures.

\section{Neural mechanism of the progesterone induced memory impairment \\ Memory encoding}

To investigate whether progesterone decreased recognition memory accuracy for faces already by modulating neural activity during encoding, brain regions that supported the successful encoding of faces were identified first. This main effect of memory was investigated by comparing encoding activity of subsequent hits and subsequent misses and showed that the amygdala, the hippocampus, and the fusiform gyrus supported memory formation of faces. In addition, regions in the bilateral inferior temporal gyri (extending to the fusiform gyri) and left middle occipital gyrus showed a larger response to subsequent hits than subsequent misses (Table 2).

Second, the main effect of drug administration showed that progesterone decreased the response of the amygdala and the fusiform gyrus while viewing the faces (i.e., faces vs null events). In contrast, progesterone increased the response in the hippocampus (Table 2). However, the progesterone administration did not significantly modulate the response in the primary visual 
Table 2. Cluster maxima for brain regions showing a main effect of successful memory encoding for faces (i.e., subsequent hits $>$ subsequent misses) or a main effect of drug administration (i.e., progesterone vs placebo) on the response to all faces (i.e., faces vs null events)

\begin{tabular}{|c|c|c|c|c|c|c|}
\hline & \multicolumn{3}{|c|}{ MNI coordinates } & \multirow[b]{2}{*}{ Cluster size } & \multirow[b]{2}{*}{$t$} & \multirow[b]{2}{*}{$p^{a}$} \\
\hline & $x$ & $y$ & $z$ & & & \\
\hline \multicolumn{7}{|c|}{ Subsequent hits $>$ subsequent misses } \\
\hline R fusiform gyrus & 48 & -52 & -16 & 109 & 5.2 & $<0.001$ \\
\hline L fusiform gyrus & -42 & -68 & -10 & 30 & 3.7 & 0.017 \\
\hline R amygdala & 24 & -4 & -22 & 19 & 3.5 & 0.013 \\
\hline L amygdala & -24 & -6 & -12 & 4 & 3.0 & 0.042 \\
\hline L hippocampus & -24 & -34 & -2 & 5 & 3.5 & 0.026 \\
\hline L middle occipital gyrus & -34 & -88 & 20 & 62 & 4.1 & $<0.001^{b}$ \\
\hline \multicolumn{7}{|l|}{ Placebo $>$ progesterone } \\
\hline R fusiform gyrus & 36 & -54 & -14 & 91 & 4.1 & 0.006 \\
\hline L amygdala & -32 & -8 & -18 & 6 & 3.0 & 0.038 \\
\hline \multicolumn{7}{|l|}{ Progesterone $>$ placebo } \\
\hline R hippocampus & 22 & -28 & -4 & 23 & 4.0 & 0.009 \\
\hline
\end{tabular}

MNI coordinates of cluster maximum are shown; the cluster size is in number of significant voxels. R, Right; L, left.

${ }^{a}$ The $p$ values are corrected for multiple comparisons across the search volume $(p<0.05)$.

${ }^{b}$ The $p$ value is uncorrected for multiple comparisons $(p<0.001 ; k \geq 20)$.
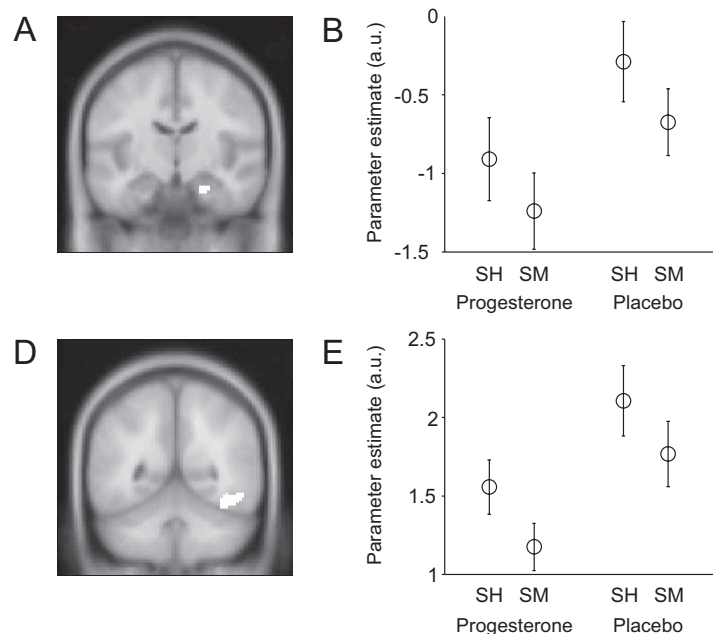
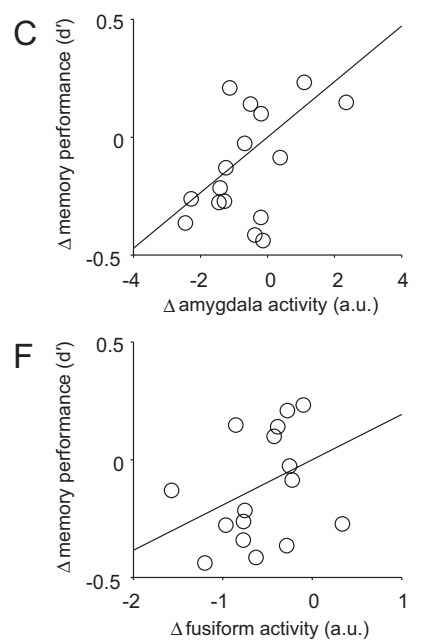

Figure 1. Progesterone reduced the recruitment of the amygdala and fusiform gyrus, brain regions that supported memory formation of faces, during memory encoding. $\boldsymbol{A}$, Significant conjunction of main effect of memory (i.e., subsequent hits $>$ subsequent misses) and main effect of drug [i.e., placebo (faces $>$ null events) $>$ progesterone (faces $>$ null events)] in the right amygdala ( $y=-12 ; p<0.001$, uncorrected). $\boldsymbol{B}$, Parameter estimates of the conjunction cluster (arbitrary units; mean \pm SEM). C, The progesterone-induced decrease in amygdala activity predicted the decrease in memory performance across subjects [recognition memory accuracy $\left(d^{\prime}\right)$ ]. $\boldsymbol{D}-\boldsymbol{F}$, Identical figures for the significant conjunction of main effects in the right fusiform gyrus ( $y=-52)$. SH, Subsequent hits; SM, subsequent misses; a.u., arbitrary units.

cortex, indicating that the drug effects in the other brain regions were not attributable to general changes in the BOLD response.

Third, a conjunction analysis was performed to confirm the spatial overlap of the main effect of memory and the main effect of drug. This analysis showed that the amygdala $[(24,-12,-22)$; $T_{(45)}^{2}=2.3 ; p=0.007$, corrected] (Fig. $1 A, B$ ), the fusiform gyrus [left $(-38,-70,-12), T_{(45)}^{2}=2.4, p=0.01$, corrected; right $(40,-52,-16), T^{2}{ }_{(45)}=3.3, p=0.036$, whole-brain corrected] (Fig. $1 D, E)$, and the middle temporal gyrus $[(56,-40$, $-12), T_{(45)}^{2}=3.7, p=0.002$, whole-brain corrected] supported memory formation and that progesterone decreased the response of these brain regions.

Fourth, to assess whether the progesterone-induced decrease in activity is related to the decrease in memory performance across subjects, the difference in activity between the progesterone and placebo sessions was entered in a regression analysis as predictor for the difference in memory performance. The de- crease in right amygdala $\left(R^{2}=0.36 ; p=\right.$ 0.005 , one-tailed) (Fig. $1 C$ ) and right fusiform gyrus $\left(R^{2}=0.30 ; p=0.012\right.$, onetailed) (Fig. $1 F$ ) activity was positively related to the decrease in $d^{\prime}$ across subjects (i.e., the extent to which progesterone modulates activity within these brain regions predicted the effect of progesterone on memory performance).

Importantly, the results showed no significant drug $\times$ memory interaction. This suggests that progesterone did not modulate memory formation per se, because progesterone did not change the differential response between subsequent hits and subsequent misses.

\section{Recognition memory}

To investigate whether progesterone decreased recognition memory accuracy also by modulating neural activity during retrieval, brain regions that supported the successful recognition of faces were identified first. The main effect of memory was investigated by comparing the recognition activity of hits and misses and showed that the fusiform gyrus also supported the recognition of faces. In addition, regions in the caudate nuclei, bilateral inferior frontal gyri, and middle cingulate gyrus showed a larger response to hits than misses (Table 3 ).

Second, as during encoding, the main effect of drug administration during recognition showed that progesterone decreased fusiform gyrus responses while subjects viewed faces (i.e., all faces vs null events). It also decreased the response in the left inferior frontal gyrus (Table 3) but did not significantly modulate the response in the primary visual cortex.

Third, the conjunction analysis showed that the fusiform gyrus [left $(-32,-64$, $-16), T_{(45)}^{2}=2.4, p=0.01$, corrected; right $(46,-56,-10), T_{(45)}^{2}=3.7, p=$ 0.005 , whole-brain corrected] (Fig. $2 A, B)$ and the inferior frontal gyrus $(p<0.001$, uncorrected; cluster, $\geq 20$ voxels) (Fig. $2 D, E$ ) supported recognition memory and that progesterone decreased the response of these brain regions.

Fourth, the regression analyses with the difference in activity between the progesterone and placebo sessions as predictor for the difference in memory performance across subjects showed that the decrease in right fusiform gyrus $\left(R^{2}=0.41 ; p=0.003\right.$, one-tailed) (Fig. $2 C)$ and left inferior frontal gyrus $\left(R^{2}=0.19\right.$; $p=0.04$, one-tailed) (Fig. $2 F$ ) activity was positively related to the decrease in $d^{\prime}$ across subjects. As during encoding, the extent to which progesterone modulated activity within these brain regions during recognition also predicted the effect of progesterone on memory performance.

Again, the drug $\times$ memory interaction showed no significant modulation of recognition processes, which suggests that progesterone did not modulate memory retrieval per se. 


\begin{tabular}{|c|c|c|c|c|c|c|}
\hline & \multicolumn{3}{|c|}{ MNI coordinates } & \multirow[b]{2}{*}{ Cluster size } & \multirow[b]{2}{*}{$t$} & \multirow[b]{2}{*}{$p^{a}$} \\
\hline & $x$ & y & $z$ & & & \\
\hline \multicolumn{7}{|l|}{ Hits $>$ misses } \\
\hline R fusiform gyrus & 46 & -56 & -10 & 44 & 4.0 & 0.008 \\
\hline R/L caudate nuclei & 14 & -2 & 16 & 479 & 4.7 & $<0.001^{b}$ \\
\hline R inferior frontal gyrus & 40 & 40 & 12 & 63 & 4.3 & $<0.001^{b}$ \\
\hline L inferior frontal gyrus & -44 & 30 & 16 & 37 & 3.7 & $<0.001^{b}$ \\
\hline Middle cingulate cortex & -4 & 2 & 34 & 55 & 3.7 & $<0.001^{b}$ \\
\hline \multicolumn{7}{|l|}{ Placebo > progesterone } \\
\hline R fusiform gyrus & 42 & -58 & -12 & 330 & 5.1 & $<0.001$ \\
\hline L fusiform gyrus & -34 & -66 & -12 & 21 & 3.5 & 0.030 \\
\hline L inferior frontal gyrus & -56 & 14 & 14 & 37 & 3.7 & $<0.001^{b}$ \\
\hline
\end{tabular}

MNI coordinates of cluster maximum are shown; the cluster size is in number of significant voxels. $R$, Right; $L$, left. ${ }^{a}$ The $p$ values are corrected for multiple comparisons across the search volume $(p<0.05)$.

${ }^{b}$ The $p$ values are uncorrected for multiple comparisons $(p<0.001 ; k \geq 20)$.

\section{Discussion}

The results of the present study demonstrate that a single progesterone administration impairs memory for faces by reducing the neural response in the amygdala and fusiform gyrus during memory encoding and in the fusiform gyrus and prefrontal cortex during memory retrieval. Moreover, the progesteroneinduced decrease in activation predicted the decrease in memory performance across subjects. However, progesterone did not affect the memory processes per se, because progesterone did not change the differential activity between (subsequent) hits and (subsequent) misses. Therefore, these results suggest that progesterone impairs memory by reducing the recruitment of those brain regions that support memory. Hence, our data reveal a neural mechanism by which progesterone affects memory for biologically salient stimuli, which links the influence of progesterone on emotion and memory. This mechanism may contribute to changes in mood regulation and cognition that are observed during the menstrual cycle and pregnancy.

Animal studies suggest that the acute effects of progesterone on memory are mediated by its neuroactive metabolite allopregnanolone, which potentiates the inhibitory actions of GABA (Majewska et al., 1986). Direct allopregnanolone administrations to rodents impair memory performance (Ladurelle et al., 2000; Johansson et al., 2002), and this learning impairment can be blocked by an allopregnanolone antagonist on the $\mathrm{GABA}_{\mathrm{A}}$ receptor (Turkmen et al., 2004). Also, human studies suggest that the progesterone-induced impairment of verbal recall is related to allopregnanolone levels (Freeman et al., 1992, 1993). However, memory effects in those studies are potentially mediated by the sedative effects of allopregnanolone, because the progesterone administrations also increased fatigue. Our results show that the effects of progesterone and/or allopregnanolone on memory are not merely a consequence of its sedative effects, because progesterone did not affect subjective (questionnaires) or objective (reaction times) measures of alertness.

The results of the present study suggest that progesterone impairs memory by reducing the recruitment of those neural processes that support memory, without affecting those processes directly. This pattern of results likely reflects the specific GABAergic action of allopregnanolone and thereby the GABAergic modulation of memory formation and retrieval. Glutamate but not GABA turnover is crucial for memory by inducing longterm potentiation (LTP) via NMDA receptor activation (Morris et al., 1986). Indeed, NMDA receptor antagonists impair human memory by affecting medial temporal lobe memory processes (Grunwald et al., 1999). In contrast, allopregnanolone reduced the response amplitude to all stimuli, regardless of whether the item was memorized or retrieved successfully. Because the activation in BOLD fMRI mainly reflects excitatory neurotransmission (Waldvogel et al., 2000), these results suggest that allopregnanolone impairs memory via a GABAergic mechanism by increasing the threshold of excitatory neurons to initiate LTP. This is likely mediated by the modulation of allopregnanolone on extrasynaptic $\delta$-subunit containing $\mathrm{GABA}_{\mathrm{A}}$ receptors, which enhances tonic inhibition and thereby decreases neuronal excitability (Stell et al., 2003). By increasing the threshold, allopregnanolone would reduce the probability that LTP is initiated. This suggests that allopregnanolone impairs memory by reducing the probability of successful memory encoding.

A similar spatial overlap of two main effects without an interaction have been observed for memory formation and divided attention (Uncapher and Rugg, 2005). Divided attention also impairs memory by reducing the probability of engaging encoding processes that support later episodic retrieval. However, divided attention also decreased the encoding efficiency in that study, because it decreased the encoding accuracy and increased reaction times. This is clearly different from the memoryimpairing effects of progesterone, because our results show no evidence of decreases in processing efficiency. It is therefore unlikely that our results reflect a progesterone-induced modulation of attention. This discrepancy with the effect of divided attention suggests that our results rather reflect a specific effect of progesterone on brain regions identified here as relevant for memory formation and retrieval.

The results suggest that progesterone impairs memory for faces by decreasing amygdala activity during memory encoding, because the amygdala supported memory formation in this study. The amygdala is essentially involved in the enhancement of memory for various emotional stimuli (Cahill et al., 1995; Richardson et al., 2004; Strange and Dolan, 2004), which suggests that the amygdala may also mediate the effect of progesterone on memory for other emotional stimuli. The memory enhancement for emotional stimuli is determined by the degree of amygdala activity and arousal during encoding (Cahill et al., 1996; Canli et al., 2000). The progesterone-induced decrease in amygdala response as observed here might thus be associated with the anxiolytic effects of allopregnanolone (Bitran et al., 1995; Akwa et al., 1999). These results therefore suggest that progesterone may impair memory by decreasing arousal.

Progesterone also decreased the neural response in the fusiform gyrus. This brain region is preferentially involved in the processing of faces (Kanwisher et al., 1997; Tsao et al., 2006), and face memory traces are potentially stored in this brain region (Miyashita, 1993; Ishai et al., 2000). These results therefore suggest that progesterone impairs memory for faces by reducing the neural response in the fusiform gyrus, which is a potential consequence of the modulation of progesterone of amygdala activity during memory encoding. The amygdala influences emotional memory by modulating memory consolidation in other brain regions (Packard et al., 1994; Kilpatrick and Cahill, 2003; McGaugh, 2004; Richardson et al., 2004) and projects back to all levels of the ventral visual stream (Amaral et al., 2003). Importantly, it modulates activity in the fusiform gyrus during face perception (Vuilleumier et al., 2004). Therefore, the progesterone induced decrease in fusiform gyrus activity may result from a decrease in modulatory influences from the amygdala. This study 

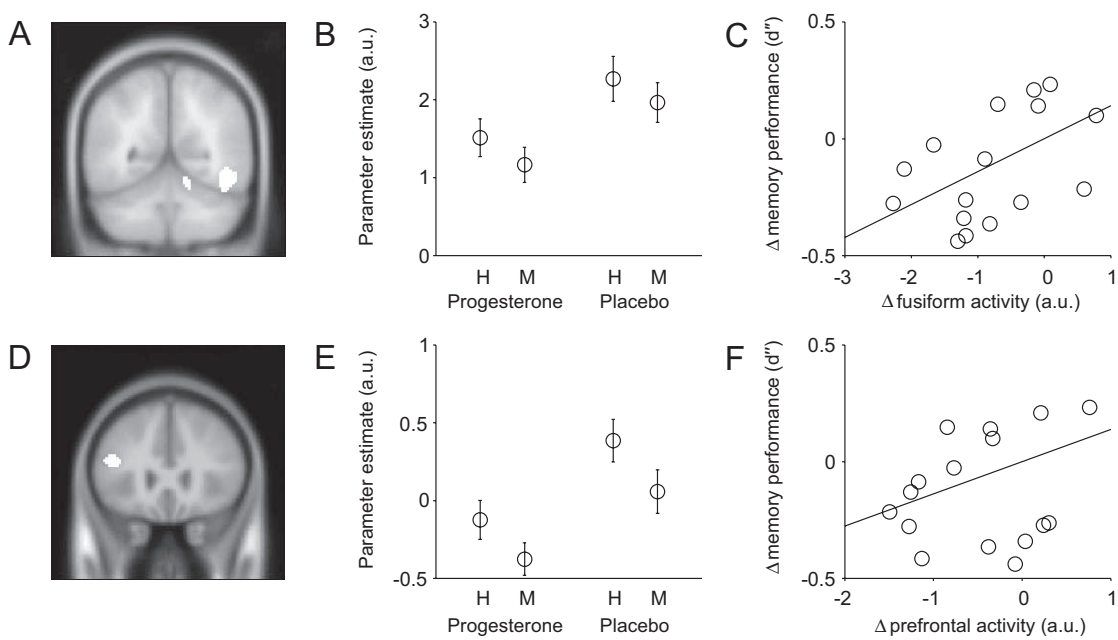

tempt to maintain adequate memory performance (Voermans et al., 2004).

Previous studies have shown that progesterone and the hormonal changes during the menstrual cycle influence amygdala activity (Goldstein et al., 2005; Dreher et al., 2007; van Wingen et al., 2007). Whereas progesterone decreased amygdala responses in the present study, progesterone can also increase amygdala activity (van Wingen et al., 2007). Although this apparent discrepancy could be attributable to differences in the experimental paradigms used, recent studies suggest that it is more likely explained by differences in allopregnanolone concentration. Whereas allopregnanolone usually decreases anxiety in animal studies (Bitran et al., 1991; Wieland et al., 1991), moderate concentrations can increase aggressive behavior (Fish et al., 2001). Moreover, women who reach moderate allopregnanolone concentrations during hormonal replacement therapy develop negative mood symptoms more often than women reaching lower and higher concentrations (Andréen et al., 2006). The paradoxical amygdala activity increase in a previous study

cannot clarify whether progesterone has an indirect effect via the amygdala or a direct effect on the fusiform gyrus, but progesterone reduced functional coupling of the amygdala with the fusiform gyrus in a blocked design with faces as stimuli (van Wingen et al., 2007). Those results suggest that the modulation of progesterone of amygdala activity influences fusiform gyrus activity, but the correlational analysis used precluded a directional interpretation. However, animal studies using amygdala lesion and drug infusion methods suggest that benzodiazepines induce their amnesic effects by decreasing (basolateral) amygdala activity (Brioni et al., 1989; Tomaz et al., 1992), likely by decreasing norepinephrine turnover within the amygdala (Hatfield et al., 1999). We therefore suggest that progesterone impairs memory by its conversion to allopregnanolone, thereby decreasing amygdala activity and its modulatory influence, which in turn downregulates memory encoding and consolidation in the fusiform gyrus.

The reduced neural responses in the inferior frontal gyrus could also contribute to the reduced fusiform gyrus responses during memory retrieval. The prefrontal cortex exerts executive control during the active retrieval of memory representations in the ITC (Tomita et al., 1999; Miyashita, 2004). The reduced prefrontal activity may therefore reflect a progesterone-induced reduction in strategic retrieval attempts. The reduction in active top-down signaling from the prefrontal cortex may subsequently reduce neural activity in the fusiform gyrus because of a reduction in search attempts.

Other human imaging studies suggest that interactions between the amygdala and the anterior hippocampus are crucial for emotional memory formation (Dolcos et al., 2004; Richardson et al., 2004). However, the results of the present study show that progesterone even increased posterior hippocampal activity while memory performance decreased. This discrepancy may be explained by the dissociation within the medial temporal lobe, because the anterior but not posterior medial temporal lobe supports emotional memory formation (Dolcos et al., 2004). The progesterone-induced hippocampal activity increase may reflect the recruitment of a compensatory neural mechanism in an at- was observed after progesterone levels had increased from the follicular to the luteal phase range (van Wingen et al., 2007), whereas progesterone levels increased to levels that are reached during pregnancy in the present study (Luisi et al., 2000). This suggests that allopregnanolone may modulate amygdala activity in a nonlinear dose-dependent manner, increasing amygdala activity at moderate concentrations, while decreasing amygdala activity at higher concentrations.

The modulation of allopregnanolone on amygdala activity may influence anxiety. Indeed, animal studies have shown that intra-amygdala infusions of allopregnanolone decreases anxiety (Akwa et al., 1999). Furthermore, inhibiting the metabolization of progesterone to allopregnanolone within the amygdala increases anxiety (Walf et al., 2006). However, a recent study indicates that allopregnanolone may modulate anxiety by modulation of the bed nucleus of the stria terminalis (BNST) rather than the amygdala. In that study, allopregnanolone attenuated corticotropin-releasing factor (CRF)-enhanced startle but not fear-potentiated startle (Toufexis et al., 2004). Previous studies have shown that CRF-enhanced startle is mediated by the BNST, whereas the amygdala mediates fear-potentiated startle. Consequently, the BNST has been proposed to support longer-lasting anxiety, and the amygdala has been proposed to support stimulus-specific fear (Walker et al., 2003). In the present study, progesterone reduced amygdala responses to specific stimuli (i.e., faces). Although our study does not exclude an effect on the BNST, the modulation of allopregnanolone on the amygdala may specifically influence fear (Walker et al., 2003) and/or modulate more generalized anxiety as suggested by other studies (Akwa et al., 1999; Walf et al., 2006).

In conclusion, we have used a placebo-controlled progesterone administration to healthy young women in combination with event-related fMRI, to provide a mechanistic account for the memory impairing effects of progesterone. Our results reveal that progesterone impairs memory for faces by reducing neural responses in the amygdala and fusiform gyrus during encoding and in the fusiform gyrus and prefrontal cortex during retrieval. The 
progesterone-induced decrease in activation predicted the decrease in memory performance across subjects. However, progesterone did not modulate the differential activation between (subsequently) remembered and (subsequently) forgotten faces. This pattern of results therefore suggests that progesterone impairs memory by reducing the recruitment of those brain regions that support memory. These mnemonic effects of progesterone are likely mediated by its neuroactive metabolite allopregnanolone. Furthermore, given the important role of the amygdala in the modulation of memory, these results suggest that allopregnanolone alters memory by influencing amygdala activity, which in turn may affect memory processes in other brain regions. These findings reveal a neural mechanism by which progesterone influences emotion and memory, which may contribute to changes in mood regulation and cognition that are observed during the menstrual cycle and pregnancy.

\section{References}

Akwa Y, Purdy RH, Koob GF, Britton KT (1999) The amygdala mediates the anxiolytic-like effect of the neurosteroid allopregnanolone in rat. Behav Brain Res 106:119-125.

Amaral DG, Behniea H, Kelly JL (2003) Topographic organization of projections from the amygdala to the visual cortex in the macaque monkey. Neuroscience 118:1099-1120.

Amunts K, Malikovic A, Mohlberg H, Schormann T, Zilles K (2000) Brodmann's areas 17 and 18 brought into stereotaxic space-where and how variable? NeuroImage 11:66-84.

Amunts K, Kedo O, Kindler M, Pieperhoff P, Mohlberg H, Shah NJ, Habel U, Schneider F, Zilles K (2005) Cytoarchitectonic mapping of the human amygdala, hippocampal region and entorhinal cortex: intersubject variability and probability maps. Anat Embryol (Berl) 210:343-352.

Andréen L, Sundström-Poromaa I, Bixo M, Andersson A, Nyberg S, Bäckström T (2005) Relationship between allopregnanolone and negative mood in postmenopausal women taking sequential hormone replacement therapy with vaginal progesterone. Psychoneuroendocrinology 30:212-224.

Andréen L, Sundström-Poromaa I, Bixo M, Nyberg S, Bäckström T (2006) Allopregnanolone concentration and mood-a bimodal association in postmenopausal women treated with oral progesterone. Psychopharmacology (Berl) 187:209-221.

Bennett HA, Einarson A, Taddio A, Koren G, Einarson TR (2004) Prevalence of depression during pregnancy: systematic review. Obstet Gynecol 103:698-709.

Bitran D, Hilvers RJ, Kellogg CK (1991) Anxiolytic effects of 3 alphahydroxy- 5 alpha $\beta$-pregnan-20-one: endogenous metabolites of progesterone that are active at the GABAA receptor. Brain Res 561:157-161.

Bitran D, Shiekh M, Mcleod M (1995) Anxiolytic effect of progesterone is mediated by the neurosteroid allopregnanolone at brain Gaba(a) receptors. J Neuroendocrinol 7:171-177.

Bond A, Lader M (1974) The use of analogue scales in rating subjective feelings. Br J Med Psychol 47:211-218.

Brett M, Baxendale S (2001) Motherhood and memory: a review. Psychoneuroendocrinology 26:339-362.

Brett M, Anton J, Valbregue R, Poline J (2002) Region of interest analysis using an SPM toolbox. Paper presented at 8th International Conference on Functional Mapping of the Human Brain, Sendai, Japan, June.

Brioni JD, Nagahara AH, McGaugh JL (1989) Involvement of the amygdala GABAergic system in the modulation of memory storage. Brain Res 487:105-112.

Buckwalter JG, Stanczyk FZ, McCleary CA, Bluestein BW, Buckwalter DK, Rankin KP, Chang L, Goodwin TM (1999) Pregnancy, the postpartum, and steroid hormones: effects on cognition and mood. Psychoneuroendocrinology 24:69-84.

Cahill L, Babinsky R, Markowitsch HJ, McGaugh JL (1995) The amygdala and emotional memory. Nature 377:295-296.

Cahill L, Haier RJ, Fallon J, Alkire MT, Tang C, Keator D, Wu J, McGaugh JL (1996) Amygdala activity at encoding correlated with long-term, free recall of emotional information. Proc Natl Acad Sci USA 93:8016-8021.

Canli T, Zhao Z, Brewer J, Gabrieli JDE, Cahill L (2000) Event-related acti- vation in the human amygdala associates with later memory for individual emotional experience. J Neurosci 20:RC99(1-5).

de Groot RH, Hornstra G, Roozendaal N, Jolles J (2003) Memory performance, but not information processing speed, may be reduced during early pregnancy. J Clin Exp Neuropsychol 25:482-488.

de Lignieres B, Dennerstein L, Backstrom T (1995) Influence of route of administration on progesterone metabolism. Maturitas 21:251-257.

Dolcos F, LaBar KS, Cabeza R (2004) Interaction between the amygdala and the medial temporal lobe memory system predicts better memory for emotional events. Neuron 42:855-863.

Dreher JC, Schmidt PJ, Kohn P, Furman D, Rubinow D, Berman KF (2007) Menstrual cycle phase modulates reward-related neural function in women. Proc Natl Acad Sci USA 104:2465-2470.

Eickhoff SB, Stephan KE, Mohlberg H, Grefkes C, Fink GR, Amunts K, Zilles K (2005) A new SPM toolbox for combining probabilistic cytoarchitectonic maps and functional imaging data. NeuroImage 25:1325-1335.

Ekman P, Friesen W (1976) Pictures of facial affect. Palo Alto, CA: Consulting Psychologist.

Fish EW, Faccidomo S, DeBold JF, Miczek KA (2001) Alcohol, allopregnanolone and aggression in mice. Psychopharmacology (Berl) 153:473-483.

Fitzgerald DA, Angstadt M, Jelsone LM, Nathan PJ, Phan KL (2006) Beyond threat: amygdala reactivity across multiple expressions of facial affect. NeuroImage 30:1441-1448.

Freeman EW, Weinstock L, Rickels K, Sondheimer SJ, Coutifaris C (1992) A placebo-controlled study of effects of oral progesterone on performance and mood. Br J Clin Pharmacol 33:293-298.

Freeman EW, Purdy RH, Coutifaris C, Rickels K, Paul SM (1993) Anxiolytic metabolites of progesterone: correlation with mood and performance measures following oral progesterone administration to healthy female volunteers. Neuroendocrinology 58:478-484.

Friston KJ, Holmes AP, Worsley KJ, Poline JB, Frith CD, Frackowiak RSJ (1995) Statistical parametric maps in functional imaging: a general linear approach. Hum Brain Mapp 2:189-210.

Friston KJ, Penny W, Phillips C, Kiebel S, Hinton G, Ashburner J (2002) Classical and Bayesian inference in neuroimaging: theory. NeuroImage 16:465-483.

Friston KJ, Penny WD, Glaser DE (2005) Conjunction revisited. NeuroImage 25:661-667.

Goldstein JM, Jerram M, Poldrack R, Ahern T, Kennedy DN, Seidman LJ, Makris N (2005) Hormonal cycle modulates arousal circuitry in women using functional magnetic resonance imaging. J Neurosci 25:9309-9316.

Grunwald T, Beck H, Lehnertz K, Blümcke I, Pezer N, Kurthen M, Fernández G, Van Roost D, Heinze HJ, Kutas M, Elger CE (1999) Evidence relating human verbal memory to hippocampal N-methyl-D-aspartate receptors. Proc Natl Acad Sci USA 96:12085-12089.

Hampson E (1990) Variations in sex-related cognitive abilities across the menstrual cycle. Brain Cogn 14:26-43.

Hatfield T, Spanis C, McGaugh JL (1999) Response of amygdalar norepinephrine to footshock and GABAergic drugs using in vivo microdialysis and HPLC. Brain Res 835:340-345.

Hochhaus L (1972) A table for the calculation of $\mathrm{d}^{\prime}$ and $\beta$. Psychol Bull 77:375-376.

Ishai A, Ungerleider LG, Haxby JV (2000) Distributed neural systems for the generation of visual images. Neuron 28:979-990.

Johansson IM, Birzniece V, Lindblad C, Olsson T, Bäckström T (2002) Allopregnanolone inhibits learning in the Morris water maze. Brain Res 934:125-131.

Kanwisher N, McDermott J, Chun MM (1997) The fusiform face area: a module in human extrastriate cortex specialized for face perception. J Neurosci 17:4302-4311.

Keenan PA, Yaldoo DT, Stress ME, Fuerst DR, Ginsburg KA (1998) Explicit memory in pregnant women. Am J Obstet Gynecol 179:731-737.

Kilpatrick L, Cahill L (2003) Amygdala modulation of parahippocampal and frontal regions during emotionally influenced memory storage. NeuroImage 20:2091-2099.

Kimura D (1999) Sex and cognition. Cambridge, MA: MIT.

Ladurelle N, Eychenne B, Denton D, Blair-West J, Schumacher M, Robel P, Baulieu E (2000) Prolonged intracerebroventricular infusion of neurosteroids affects cognitive performances in the mouse. Brain Res 858:371-379.

Luisi S, Petraglia F, Benedetto C, Nappi RE, Bernardi F, Fadalti M, Reis FM, 
Luisi M, Genazzani AR (2000) Serum allopregnanolone levels in pregnant women: changes during pregnancy, at delivery, and in hypertensive patients. J Clin Endocrinol Metab 85:2429-2433.

Majewska MD, Harrison NL, Schwartz RD, Barker JL, Paul SM (1986) Steroid hormone metabolites are barbiturate-like modulators of the GABA receptor. Science 232:1004-1007.

Maki PM, Rich JB, Rosenbaum RS (2002) Implicit memory varies across the menstrual cycle: estrogen effects in young women. Neuropsychologia 40:518-529.

McGaugh JL (2004) The amygdala modulates the consolidation of memories of emotionally arousing experiences. Annu Rev Neurosci 27:1-28.

Miyashita Y (1993) Inferior temporal cortex: where visual perception meets memory. Annu Rev Neurosci 16:245-263.

Miyashita Y (2004) Cognitive memory: cellular and network machineries and their top-down control. Science 306:435-440.

Morris RG, Anderson E, Lynch GS, Baudry M (1986) Selective impairment of learning and blockade of long-term potentiation by an N-methyl-Daspartate receptor antagonist, AP5. Nature 319:774-776.

N-Wihlbäck AC, Sundström-Poromaa I, Bäckström T (2006) Action by and sensitivity to neuroactive steroids in menstrual cycle related CNS disorders. Psychopharmacology (Berl) 186:388-401.

Packard MG, Cahill L, McGaugh JL (1994) Amygdala modulation of hippocampal-dependent and caudate nucleus-dependent memory processes. Proc Natl Acad Sci USA 91:8477-8481.

Phillips SM, Sherwin BB (1992) Variations in memory function and sex steroid hormones across the menstrual cycle. Psychoneuroendocrinology 17:497-506.

Richardson MP, Strange BA, Dolan RJ (2004) Encoding of emotional memories depends on amygdala and hippocampus and their interactions. Nat Neurosci 7:278-285.

Schmidt PJ, Nieman LK, Danaceau MA, Adams LF, Rubinow DR (1998) Differential behavioral effects of gonadal steroids in women with and in those without premenstrual syndrome. N Engl J Med 338:209-216.

Schmitt JA, Jorissen BL, Dye L, Markus CR, Deutz NE, Riedel WJ (2005) Memory function in women with premenstrual complaints and the effect of serotonergic stimulation by acute administration of an alphalactalbumin protein. J Psychopharmacol 19:375-384.

Sharp K, Brindle PM, Brown MW, Turner GM (1993) Memory loss during pregnancy. Br J Obstet Gynaecol 100:209-215.

Sheehan DV, Lecrubier Y, Sheehan KH, Amorim P, Janavs J, Weiller E, Hergueta T, Baker R, Dunbar GC (1998) The Mini-International Neuropsychiatric Interview (M.I.N.I.): the development and validation of a structured diagnostic psychiatric interview for DSM-IV and ICD-10. J Clin Psychiatry 59 [Suppl 20]:22-33.

Somerville LH, Kim H, Johnstone T, Alexander AL, Whalen PJ (2004) Human amygdala responses during presentation of happy and neutral faces: correlations with state anxiety. Biol Psychiatry 55:897-903.

Spielberger CD, Gorsuch RL, Lushene RE (1970) STAI manual for the State Trait Anxiety Inventory. Palo Alto, CA: Consulting Psychologists.

Steiner M, Dunn E, Born L (2003) Hormones and mood: from menarche to menopause and beyond. J Affect Disord 74:67-83.

Stell BM, Brickley SG, Tang CY, Farrant M, Mody I (2003) Neuroactive steroids reduce neuronal excitability by selectively enhancing tonic inhibition mediated by delta subunit-containing GABAA receptors. Proc Natl Acad Sci USA 100:14439-14444.
Strange BA, Dolan RJ (2004) Beta-adrenergic modulation of emotional memory-evoked human amygdala and hippocampal responses. Proc Natl Acad Sci USA 101:11454-11458.

Sveindottir H, Backstrom T (2000) Prevalence of menstrual cycle symptom cyclicity and premenstrual dysphoric disorder in a random sample of women using and not using oral contraceptives. Acta Obstet Gynecol Scand 79:405-413.

Timby E, Balgard M, Nyberg S, Spigset O, Andersson A, PorankiewiczAsplund J, Purdy RH, Zhu D, Backstrom T, Poromaa IS (2006) Pharmacokinetic and behavioral effects of allopregnanolone in healthy women. Psychopharmacology (Berl) 186:414-424.

Tomaz C, Dickinson-Anson H, McGaugh JL (1992) Basolateral amygdala lesions block diazepam-induced anterograde amnesia in an inhibitory avoidance task. Proc Natl Acad Sci USA 89:3615-3619.

Tomita H, Ohbayashi M, Nakahara K, Hasegawa I, Miyashita Y (1999) Topdown signal from prefrontal cortex in executive control of memory retrieval. Nature 401:699-703.

Toufexis DJ, Davis C, Hammond A, Davis M (2004) Progesterone attenuates corticotropin-releasing factor-enhanced but not fear-potentiated startle via the activity of its neuroactive metabolite, allopregnanolone. J Neurosci 24:10280-10287.

Tsao DY, Freiwald WA, Tootell RB, Livingstone MS (2006) A cortical region consisting entirely of face-selective cells. Science 311:670-674.

Turkmen S, Lundgren P, Birzniece V, Zingmark E, Backstrom T, Johansson IM (2004) 3beta-20beta-dihydroxy-5alpha-pregnane (UC1011) antagonism of the GABA potentiation and the learning impairment induced in rats by allopregnanolone. Eur J Neurosci 20:1604-1612.

Uncapher MR, Rugg MD (2005) Effects of divided attention on fMRI correlates of memory encoding. J Cogn Neurosci 17:1923-1935.

van Wingen GA, van Broekhoven F, Verkes RJ, Petersson KM, Bäckström T, Buitelaar JK, Fernández G (2007) Progesterone selectively increases amygdala reactivity in women. Mol Psychiatry, in press.

Voermans NC, Petersson KM, Daudey L, Weber B, Van Spaendonck KP, Kremer HP, Fernandez G (2004) Interaction between the human hippocampus and the caudate nucleus during route recognition. Neuron 43:427-435.

Vuilleumier P, Richardson MP, Armony JL, Driver J, Dolan RJ (2004) Distant influences of amygdala lesion on visual cortical activation during emotional face processing. Nat Neurosci 7:1271-1278.

Waldvogel D, van Gelderen P, Muellbacher W, Ziemann U, Immisch I, Hallett M (2000) The relative metabolic demand of inhibition and excitation. Nature 406:995-998.

Walf AA, Sumida K, Frye CA (2006) Inhibiting 5alpha-reductase in the amygdala attenuates antianxiety and antidepressive behavior of naturally receptive and hormone-primed ovariectomized rats. Psychopharmacology (Berl) 186:302-311.

Walker DL, Toufexis DJ, Davis M (2003) Role of the bed nucleus of the stria terminalis versus the amygdala in fear, stress, and anxiety. Eur J Pharmacol 463:199-216.

Wieland S, Lan NC, Mirasedeghi S, Gee KW (1991) Anxiolytic activity of the progesterone metabolite 5 alpha-pregnan-3 alpha-o1-20-one. Brain Res 565:263-268.

Worsley KJ, Marrett S, Neelin P, Vandal AC, Friston KJ, Evans AC (1996) A unified statistical approach for determining significant signals in images of cerebral activation. Hum Brain Mapp 4:58-73. 